\title{
Localisation in focal epilepsy: a practical guide
}

\author{
Fahmida A Chowdhury (D) , ${ }^{1,2}$ Rui Silva, ${ }^{1}$ Benjamin Whatley, ${ }^{1,3}$ \\ Matthew C Walker ${ }^{1,2}$
}

- Additional online supplemental material is published online only. To view, please visit the journal online (http://dx.doi.org/10.1136/ practneurol-2019-002341).

'Department of Epilepsy, National Hospital for Neurology and Neurosurgery, London, UK ${ }^{2}$ Department of Clinical and Experimental Epilepsy, Institute of Neurology, London, UK ${ }^{3}$ Department of Neurology, Dalhousie University, Halifax, Nova Scotia, Canada

\section{Correspondence to \\ Fahmida A Chowdhury, National Hospital for Neurology and Neurosurgery, London WC1N 3BG, UK; fahmidaamin. chowdhury@nhs.net}

Accepted 26 May 2021 Published Online First 17 August 2021

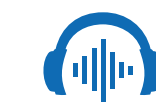

Listen to Podcast pn.bmj.com

Check for updates

(C) Author(s) (or their employer(s)) 2021. No commercial re-use. See rights and permissions. Published by BMJ.

To cite: Chowdhury FA Silva $\mathrm{R}$, Whatley $\mathrm{B}$, et al. Pract Neurol 2021;21:481491.

\begin{abstract}
The semiology of epileptic seizures reflects activation, or dysfunction, of areas of brain (often termed the symptomatogenic zone) as a seizure begins and evolves. Specific semiologies in focal epilepsies provide an insight into the location of the seizure onset zone, which is particularly important for presurgical epilepsy assessment. The correct diagnosis of paroxysmal events also depends on the clinician being familiar with the spectrum of semiologies. Here, we summarise the current literature on localisation in focal epilepsies using illustrative cases and discussing possible pitfalls in localisation.
\end{abstract}

\section{INTRODUCTION}

The clinical signs (semiology) of seizures are the manifestation of epilepsy due to altered electrical activity in the affected part of the brain; these phenomena can be motor, behavioural or subjective, with or without altered consciousness. Hughlings Jackson recognised this over 150 years ago and in 1868 described the characteristic 'Jacksonian march'-seen in focal motor seizures arising from primary motor cortex-writing that 'there will be all varieties of epilepsy, according to the exact position of grey matter altered'. ${ }^{1}$ Careful analysis of semiology, using both the patient's history and data from videotelemetry, can help to localise seizure onset. This is particularly important in presurgical epilepsy assessment, something that should be considered for everyone with medically refractory focal epilepsies. Clinicians also need to be familiar with the spectrum of epilepsy semiologies in order to make the correct diagnosis and to differentiate epileptic seizures from dissociative events, syncope and other non-epileptic events. The symptomatogenic zone is where symptoms and clinical signs originate; it is usually, but not always, close to the epileptogenic zone, which is the part of the brain that must be removed to render the patient seizure free, and is the objective of epilepsy surgery. ${ }^{2}$ Table 1 describes further these and other relevant concepts in presurgical assessment. Electrical stimulation studies have helped to identify regions of brain involved in generating different types of semiology; however, these also show that most of cortex is clinically silent, and clinical signs and symptoms occur only when the seizure spreads to activate or disrupt cortical networks. ${ }^{3}$

Clinical signs evolve as the seizure propagates, and reflect the anatomical sites involved in its spread. However, seizure propagation comprises two distinct phenomena. ${ }^{4}$ There is an ictal wavefront moving at about $1 \mathrm{~mm} / \mathrm{s}$ as neurones are slowly recruited into firing; this represents, for example, the speed of the Jacksonian march with slow tangential activation of cortex. There is also a much faster ictal discharge spreading at about $300 \mathrm{~mm} / \mathrm{s}$, which represents the propagation of excitation/inhibition into neighbouring or connected brain areas. It is this faster propagation that electroencephalogram (EEG) electrodes detect. Thus, an area seen to be recruited into ictal activity on EEG is not necessarily firing, since we are seeing the input to that area but not its behaviour. The seizure semiology can, therefore, be generated by both activation of brain areas, which occurs slowly, and by the disruption of brain networks, which occurs more rapidly. Propagation of seizure activity and semiology is further complicated by the recruitment of subcortical areas, leading to rapid engagement of widespread areas of cortex. It is therefore not surprising that the rate of a seizure's progression depends on several factors, foremost among which is localisation. For example, temporal lobe seizures, which depend mainly on activation of brain areas, evolve more slowly, whereas 
Table 1 Cortical zones defined in presurgical evaluation

\begin{tabular}{ll}
\hline Cortical zone & Definition \\
\hline $\begin{array}{l}\text { Symptomatogenic zone } \\
\text { Seizure onset zone }\end{array}$ & $\begin{array}{l}\text { The area of cortex which, when activated by an epileptiform discharge, produces the initial ictal symptoms; it is usually } \\
\text { an area of eloquent cortex, and may be outside the epileptogenic zone. }\end{array}$ \\
$\begin{array}{l}\text { The area of cortex from which clinical seizures actually originate. } \\
\text { Trritative zone }\end{array}$ & $\begin{array}{l}\text { The area of cortex that is necessary for the generation of seizures, and must be removed (or disconnected) to render } \\
\text { patients seizure-free after surgery; it may be larger than the seizure onset zone and the epileptogenic lesion. }\end{array}$ \\
Epileptogenic lesion & The macroscopic lesion seen on the MR scan that is causing the epileptic seizures. \\
\hline
\end{tabular}

frontal lobe seizures, which may depend on disinhibition, propagate quickly. While certain semiologies, in particular those that involve activation of eloquent brain areas, are pathognomonic (such as focal motor seizures that arise from contralateral primary motor cortex) other signs are of less localising value, for example, automotor seizures that can arise from different brain regions.

Before there were EEGs, clinical semiology analysis relied mainly on postmortem studies. Though EEG enables mapping of the ictal onset and spread, it does not tell us about the behaviour of the underlying cortex when such activity is detected. EEG has further limitations, for example, with seizures arising from deep sources such as orbitofrontal cortex, where there may be no or widespread EEG changes at the scalp; intracranial recordings can overcome this to some extent. Advances in neuroimaging have also allowed better precision in localising semiology. However, the epileptic region may involve relatively normal brain adjacent to a lesion, and some lesions may be incidental. In addition, ictal manifestations may represent propagation of epileptic activity from its origin, and therefore, the clinician needs to integrate all the available data (history, examination, neuroimaging, neurophysiology and neuropsychology) to determine the epileptogenic zone. However, seizure semiology should certainly not be disregarded. In a recent study looking at a series of patients successfully treated with epilepsy surgery, ${ }^{5}$ seizure semiology was correctly lateralising in $74 \%$ and correctly localising in $77 \%$ of patients, on a par with scalp EEG and MRI.

In this paper, we summarise the current literature on localisation of focal seizures and discuss some of its complexities and possible pitfalls. We have also made available case examples from our centre as online supplemental videos. There is extensive literature on this topic and so we have focused on the most common semiologies.

\section{FRONTAL LOBE SEIZURES}

The frontal lobe is the largest lobe of the brain. Its high connectivity with other cortical regions can result in rapid, widespread ictal propagation. Moreover, some of the semiological features from more rostral areas may result more from disinhibition (removal of higher cortical control) than from direct activation. Together, these can make frontal lobe seizure semiology complex and difficult to interpret. Nevertheless, seizures have characteristic patterns depending on the area of the frontal lobe involved. In general, frontal lobe seizures are typically brief, may cluster, can have prominent vocalisation and commonly occur from sleep. Motor features are prominent and include various different motor phenomena, including simple clonic movements, tonic posturing and eye deviation, or more complex movements such as cycling, rocking and grimacing. Seizures can appear bizarre, with bilateral motor phenomena, but with retained awareness. Ictal scalp EEG may show no changes if there is a deep source, or may be obscured by movement artefact, and so frontal lobe seizures may be mistaken for dissociative (non-epileptic) attacks. Figure 1 summarises the semiology of seizures arising from the frontal lobe by region.

The ictal onset patterns during intracranial studies with stereo-EEG ${ }^{67}$ show that elementary motor signs (clonic, tonic, versive) arise from precentral and premotor regions, while more complex motor signs arise from more rostral frontal regions. Non-integrated (less natural) gestural behaviour with proximal stereotypies, such as rocking and turning, are from premotor and posterior prefrontal regions, and more integrated (natural) gestural behaviour with distal stereotypies, such as manual automatisms, arise from more anterior prefrontal regions (orbitofrontal cortex, frontal pole, anterior cingulate). In clinical practice, these distinctions may be confounded by rapid propagation.

\section{Primary motor cortex}

Seizures from primary motor cortex comprise contralateral unilateral clonic or sometimes tonic or myoclonic activity that can spread to adjacent areas: the 'Jacksonian march', reflecting ictal activity spread through the motor cortex (video 1) along the homunculus. It is therefore possible to distinguish lateral from medial precentral seizures, depending on whether the seizure starts in face, upper or lower limb. ${ }^{8}$ The spread is typically slow, representing the slow recruitment 


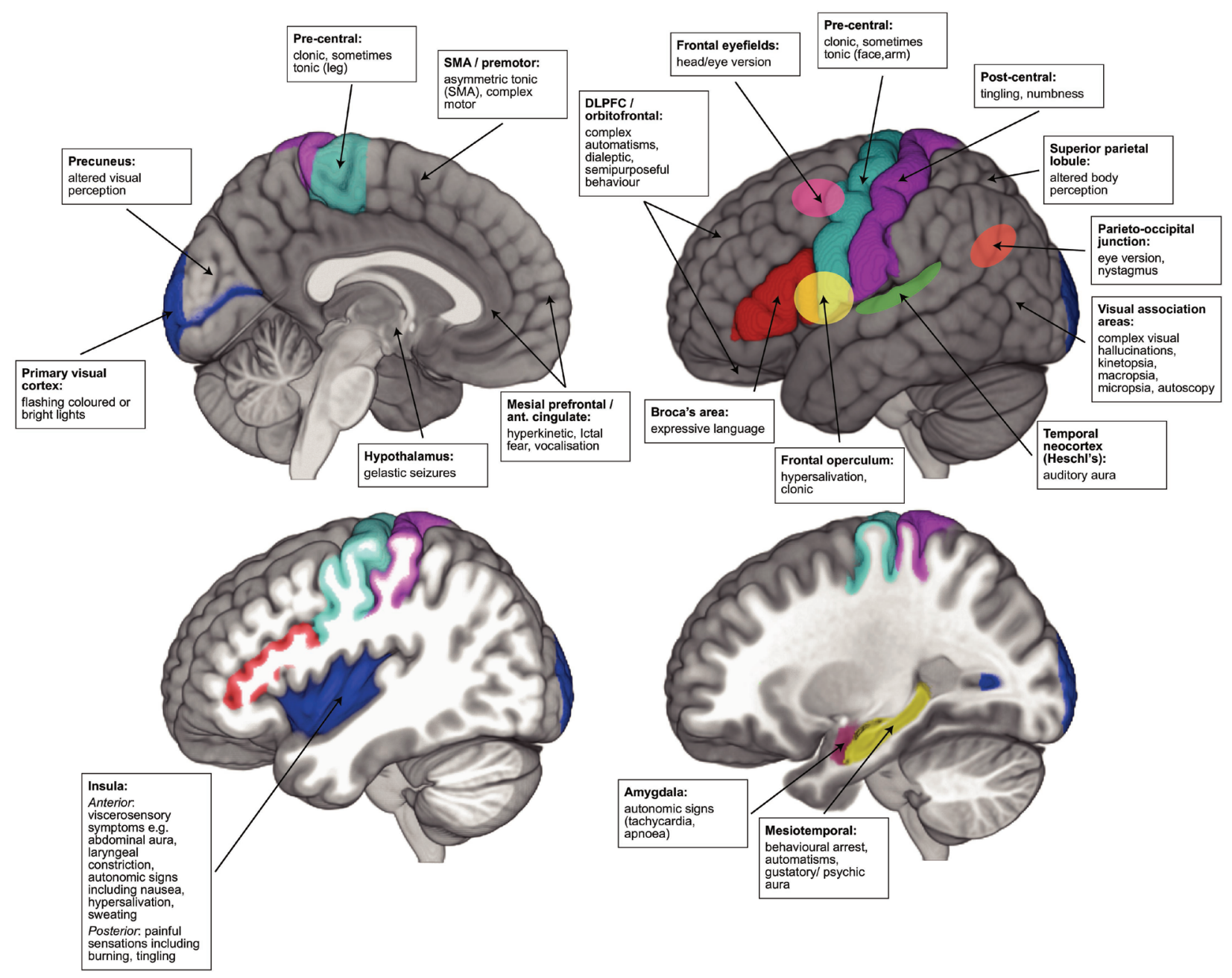

Figure 1 Summary of semiological signs by symptomatogenic zone on mesial and lateral structures. DLPFC, dorsolateral prefrontal cortex; SMA, supplementary motor area.

of neurones during tangential spread of the ictal wavefront.

Video case 1: A 50-year-old man with seizure onset at aged 28 reported ongoing focal to bilateral tonicclonic seizures. MR scan of brain showed a cystic lesion in the right middle frontal gyrus (figure 2: T1 MRI). Videotelemetry captured a seizure that began with an

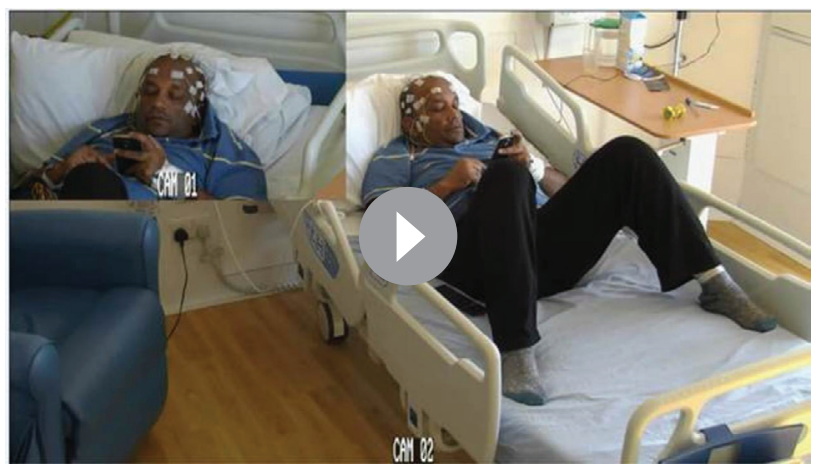

Video 1 Video case 1. aura (he is seen reaching for the button but later did not recall the aura), followed by behavioural arrest, and clonic movements visible in the left face spreading in a Jacksonian fashion over the left arm as the seizure spreads over the right primary motor cortex, followed by left arm extension (fencing posture) indicating involvement of supplementary motor area, before secondary generalisation. The semiology pointed to right hemispheric onset and ictal EEG showed onset in the right frontocentral region, in keeping with the lesion.

\section{Supplementary motor area}

Seizures arising in the supplementary motor area (SMA) are characterised by asymmetric bilateral tonic posturing, with or without impaired awareness. There may be extension of the upper limb contralateral to the hemisphere of onset, and flexion of the ipsilateral upper limb, known as a 'fencing posture' (video 1), along with head and eye deviation contralateral to the hemisphere of onset. The motor features can be preceded by sensory features such as tingling, or 


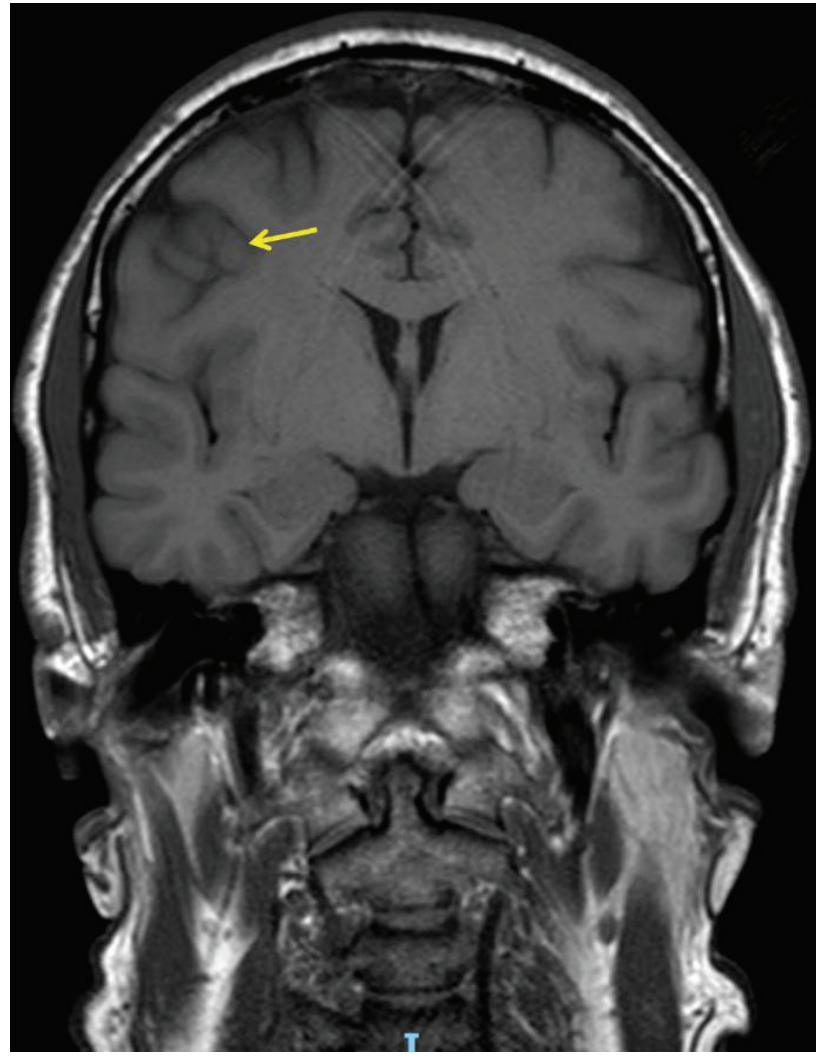

Figure 2 Case 1: T1 MR scan of brain showing lesion in right middle frontal gyrus (shown with arrow).

tightness. Since the supplementary motor area is highly connected to other parts of the brain, seizures arising from other regions (especially parietal and occipital lobe seizures) can have asymmetric tonic posturing. ${ }^{9}$ However, seizures arising from extrafrontal regions are more likely to be preceded by auras or to involve loss of awareness. ${ }^{10} 11$

\section{Other frontal regions}

Seizures arising from orbitofrontal cortex tend to have impaired awareness and automatisms, ${ }^{12}$ and may evolve to complex motor seizures (video 2). Olfactory auras occasionally occur and there may be autonomic features such as ictal tachycardia. Seizures arising from frontopolar cortex can also

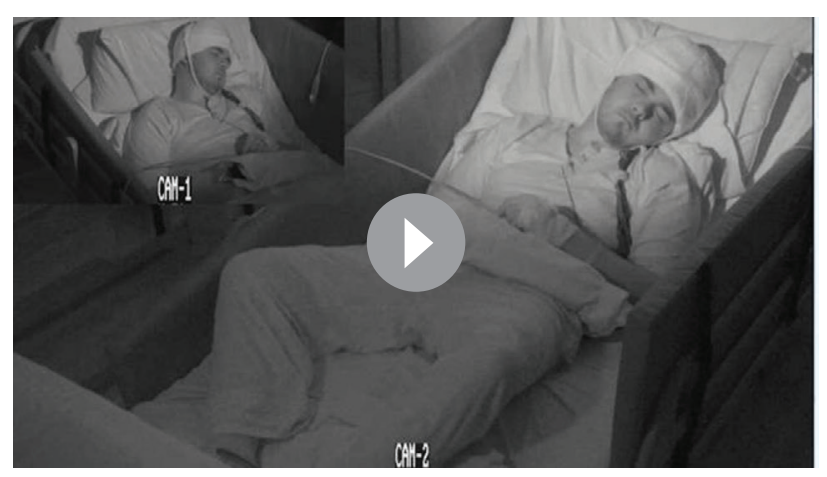

Video 2 Video case 2. start with impaired awareness and progress to show other motor features. Seizures arising from ventromedial prefrontal regions, including anterior cingulate, have been associated with 'forced thinking' and have hyperkinetic features; patients may appear to be frightened. ${ }^{6}$ Patients with seizures arising from the contralateral dorsolateral frontal cortex (involving the frontal eye fields) may show early head version (forced, sustained and extreme turning of the head with neck extension) and eye version. ${ }^{7}$ Seizures arising in the dominant hemisphere, especially if in or near Broca's area, can cause dysphasia. Seizures arising from frontal operculum are characterised by face clonic movements that may be unilateral, with laryngeal symptoms, hypersalivation and articulation difficulties. Spread to the insula means there is significant overlap of symptoms with seizures arising from the insula (see later). Focal atonic seizures can occur in frontal lobe epilepsies and may indicate involvement of negative motor areas, ${ }^{13}$ or the supplementary motor area. ${ }^{14}$ Gelastic seizures and ictal pouting may also occur in frontal lobe seizures (mesiofrontal and anterior cingulate) but we discuss these separately below, along with lateralising signs.

Video case 2: A 24-year-old man developed hyperkinetic seizures from sleep. MR scan of brain was non lesional, and positron emission tomography (PET) scan showed hypometabolism in the right orbitofrontal region (figure 3). During an intracranial implantation with stereo-EEG, we captured habitual hyperkinetic seizures that progressed to bilateral tonic seizures with loss of awareness. The EEG onset was seen in the right orbitofrontal region, and there were tonic features when the EEG seizure spread to electrode contacts in the supplementary motor area. Following resection of the right orbitofrontal region he is now seizure free.

\section{TEMPORAL LOBE SEIZURES}

The temporal lobes are the most frequent site of origin of focal seizures; two thirds of people with refractory epilepsy assessed for potential surgery have temporal lobe seizures. They typically comprise behavioural arrest, manual and oral automatisms in two thirds, and variable degrees of loss of awareness with postictal confusion ${ }^{15}$ (see figure 1 for a summary). Though there is some overlap with frontal lobe seizures, temporal lobe seizures tend to be longer (up to 2-3 min) and less frequent, with longer periods of postictal confusion. Table 2 shows other differentiating factors. Focal impaired awareness seizures arising from the temporal lobe can be distinguished clinically from generalised 'absence' seizures that occur in the genetic (idiopathic) generalised epilepsies. Absences are typically very short periods of motor arrest, lasting seconds, without preceding aura or postictal confusion and occur many times a day. In comparison, focal impaired awareness 


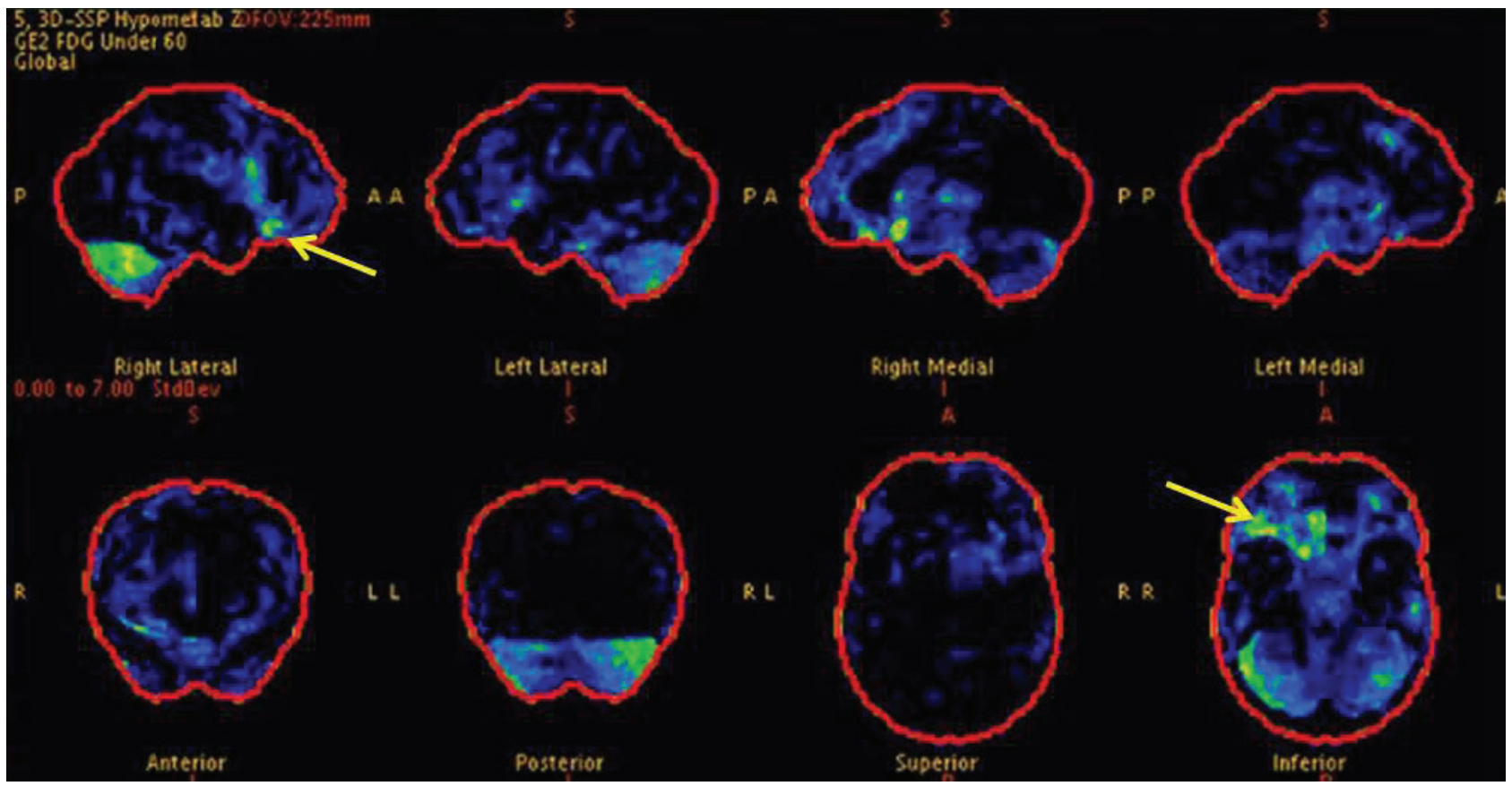

Figure 3 Case 2: Positron emission tomography (PET) scan of brain showing hypermetabolism in right orbitofrontal region.

seizures are longer, may be preceded by aura and are commonly associated with automatisms, described below in more detail.

\section{Auras}

Auras occur commonly in temporal lobe seizures; some are relatively specific. For example an abdominal aura (such as rising epigastric sensation or abdominal discomfort) is highly associated with temporal lobe epilepsy with a probability of $74 \%$, and the evolution of an abdominal aura into an automotor seizure increases the probability of temporal lobe epilepsy

Table 2 Temporal and frontal lobe seizures differentiating semiological features.

\begin{tabular}{|c|c|c|}
\hline Features & Frontal & Temporal \\
\hline Seizure frequency & Often daily & Less frequent \\
\hline Occurrence during sleep & Typical & Less common \\
\hline Seizure onset & Abrupt & Gradual \\
\hline Seizure duration & $\begin{array}{l}\text { Brief (usually up to } \\
30 \mathrm{~s})^{*}\end{array}$ & $\begin{array}{l}\text { Longer (usually } 30 \\
\text { s to } 2 \mathrm{~min})^{*}\end{array}$ \\
\hline Evolution & Rapid & Gradual \\
\hline Automatisms & Less common & $\begin{array}{l}\text { Common and } \\
\text { longer }\end{array}$ \\
\hline Complex motor features & $\begin{array}{l}\text { Early, frequent, } \\
\text { prominent }\end{array}$ & Late, less frequent \\
\hline Hypermotor & Common & Rare \\
\hline Vocalisation & $\begin{array}{l}\text { Loud, nonspeech } \\
\text { (scream, grunt) }\end{array}$ & $\begin{array}{l}\text { Ictal speech } \\
\text { (nondominant) }\end{array}$ \\
\hline Secondary generalisation & Common & Less common \\
\hline Postictal confusion & $\begin{array}{l}\text { Shorter, less } \\
\text { prominent }\end{array}$ & $\begin{array}{l}\text { Longer, more } \\
\text { prominent }\end{array}$ \\
\hline
\end{tabular}

${ }^{*}$ Can be longer. to $98 \% .{ }^{16}$ Gustatory and olfactory hallucinations are also relatively specific for temporal lobe epilepsy. Somatosensory auras can occur and usually manifest as either unilateral or bilateral tingling. ${ }^{17}$ The type of aura can help to differentiate between seizures of mesiotemporal origin vs lateral neocortical origin. In a large study of 187 temporal lobe seizures assessed by intracranial stereo-EEG, abdominal aura and experiential phenomena such as fear, déjà vu and jamais vu indicated mesiotemporal seizures, whereas elemental auditory aura indicated lateral onset (Heschl's gyrus, primary auditory cortex). ${ }^{18}$

\section{Mesial temporal lobe including hippocampus}

Seizures arising in the mesial temporal lobe may have the experiential aura described above, but also early autonomic phenomena such as flushing or tachycardia. Early apnoea and tachycardia indicate involvement of amygdala. ${ }^{19}$ These symptoms may progress to behavioural arrest, automatisms, and impaired

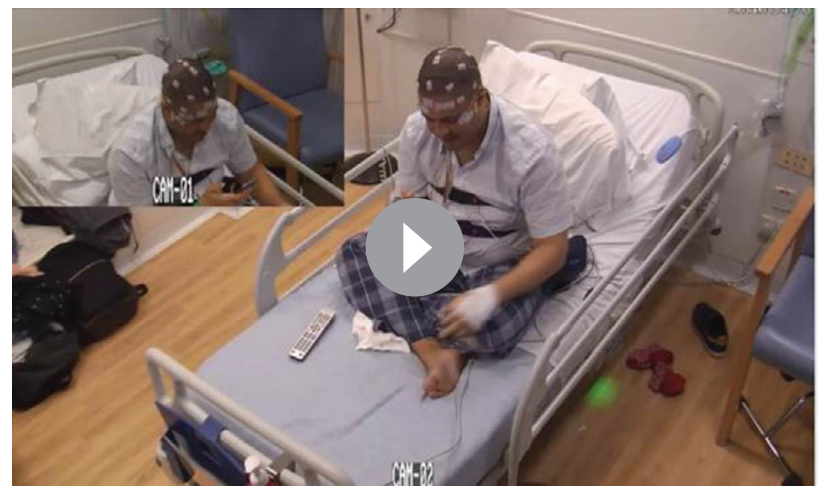

Video 3 Video case 3. 


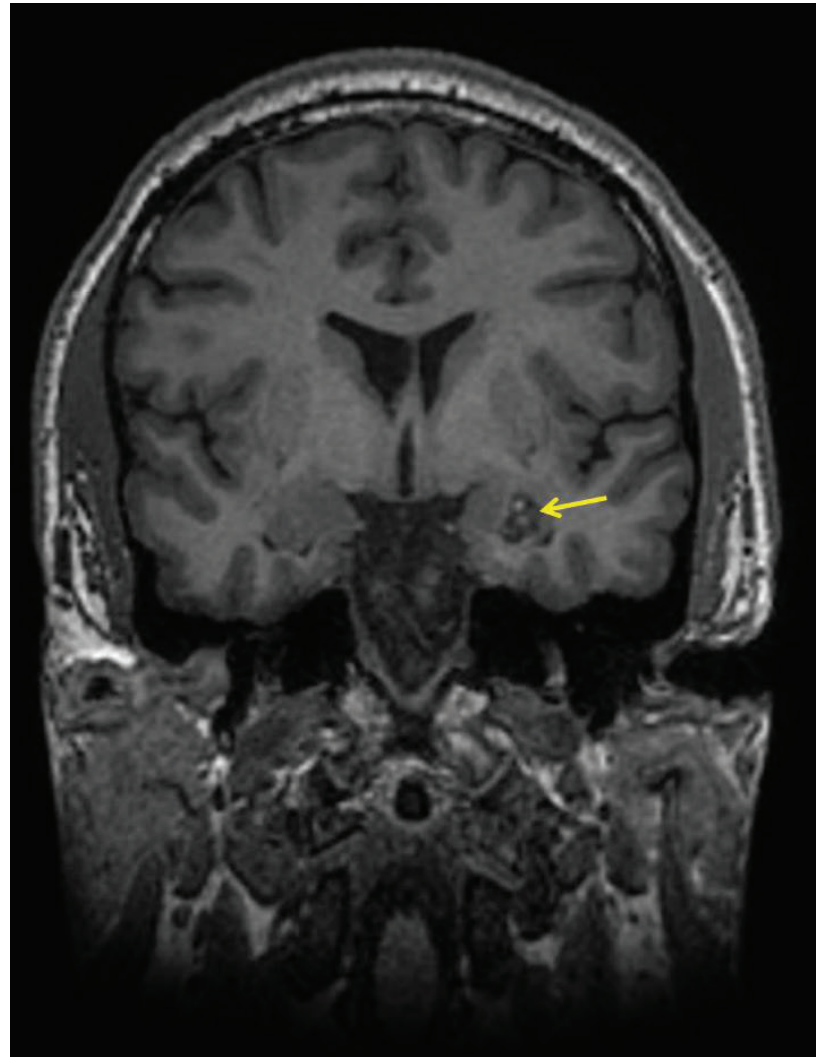

Figure 4 Case 3: T1 MR scan of brain showing cavernoma in left mesiotemporal region (shown with arrow).

awareness. Automatisms often involve the hands (fumbling, picking, fidgeting) or mouth (chewing, lip smacking, swallowing) (video 3). Less common automatisms associated with temporal lobe seizures include vocalisations and ictal speech (in non-dominant seizures). Unilateral dystonic posturing can occur and is a contralateral sign. ${ }^{20}$ Seizures tend to be longer that those arising from lateral temporal lobe, and they less commonly evolve to bilateral tonic-clonic seizures. ${ }^{21}$

Video case 3: A 36-year-old right-handed man had seizure onset at the age of 21 , characterised by déjà vu and epigastric rising, evolving to automotor features, with rare focal to bilateral tonic-clonic seizures. Imaging showed a cavernoma in the left amygdala and hippocampal head (figure 4). Videotelemetry captured habitual seizures with EEG confirming onset in the left temporal region. Seizures had typical features of mesiotemporal lobe seizures, with psychic and abdominal aura, followed by oral and manual automatisms, then loss of awareness. Before the seizure generalised, there was head and eye version to the right along with a 'figure-of-4' sign (left arm flexed and right arm extended), which lateralised the onset to the left hemisphere. He subsequently underwent a lesionectomy and remains seizure free.

\section{Lateral/neocortical temporal lobe}

Auras are less common in seizures arising from temporal neocortex, and often have auditory or vertiginous

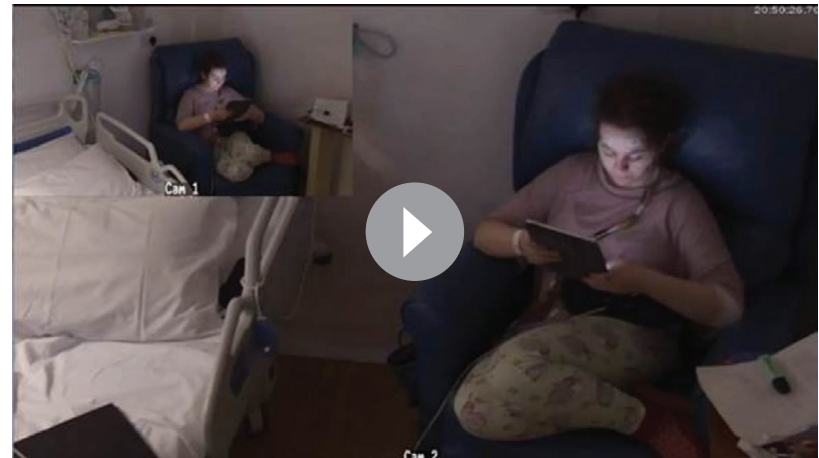

Video 4 Video case 4

features (temporoparietal junction). Complex visual hallucination and illusions can occur with anteromedial and temporo-occipital lobe seizures, but often do not clearly distinguish neocortical from mesial temporal onset. The most common auditory aura is of elementary sounds (humming, ringing, buzzing) indicating involvement of primary auditory cortex, and less frequently complex sounds (voices, songs) or distortions, indicating involvement of auditory association areas. Elementary unilateral auditory aura indicates onset in contralateral hemisphere. Ictal dysphasia may be an early sign of dominant hemisphere involvement (video 4). Lateral temporal seizures are shorter than mesial temporal lobe seizures, with earlier loss of awareness and propagation to frontal regions resulting in complex motor features and more common evolution to a bilateral tonic-clonic seizure. ${ }^{18}$ However, due to reciprocal connections between mesial and neocortical temporal cortex, the semiology of these seizures overlaps.

Video case 4: A 27-year-old woman had seizure onset at age 18 years, which would begin with a psychic aura of fear and anxiety and sometimes a noise in her ears, followed by difficulty speaking. MR scan of brain was non-lesional and PET scan showed hypometabolism in the left temporal region (figure 5), which on statistical analysis pointed to left superior temporal gyrus extending to temporo-parieto-occipital junction. Videotelemetry captured stereotypical seizures with ictal dysphasia. Assessment by nurses showed she could engage and follow visual commands but not verbal commands, with impairment of both expressive language and reading. Ictal EEG showed onset in the left temporal region suggesting a neocortical onset.

\section{Temporal pole seizures}

Temporal pole seizures tend to have earlier loss of consciousness $^{21}$ and more commonly present with hypermotor features. ${ }^{22}$

\section{PARIETAL LOBE SEIZURES}

Parietal lobe epilepsy is rare, accounting for 5\% of epilepsies. ${ }^{23}$ However, seizures arising from the parietal lobe can have diverse semiology and can be 


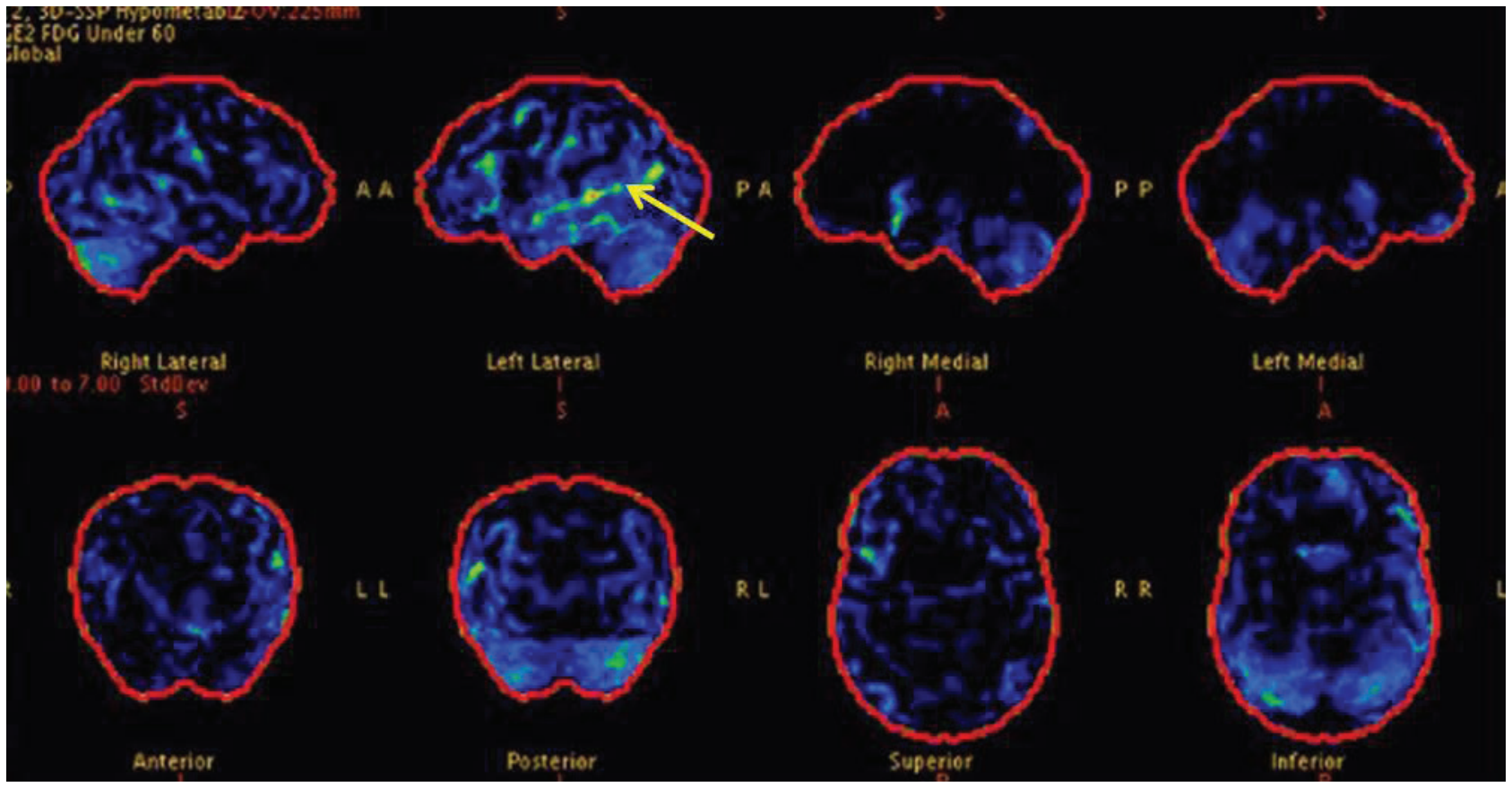

Figure 5 Case 4: PET scan of brain showing hypometabolism in left temporal lobe (shown with arrow).

difficult to diagnose due to their subjective nature. In addition, the rich connections to adjacent structures, within and outside the parietal lobe, means that the symptomatogenic zone may be distant to the seizureonset zone, and therefore, parietal lobe epilepsy may be both misdiagnosed and also underdiagnosed. Epilepsy surgery is least commonly performed on the parietal lobe, and so there are few data on seizure localisation. In two of the larger surgical series of patients with parietal lobe epilepsy, ${ }^{24} 25$ the most commonly reported aura (in 38\% in both series) was contralateral somatosensory aura. Other subjective reported phenomena of parietal lobe epilepsy include vertiginous aura, distortion of body image or visual perception. There may be auditory, olfactory and epigastric aura, suggesting propagation to temporal and limbic areas.

\section{Primary sensory cortex}

Seizures arising from the primary sensory cortex in the postcentral gyrus tend to start with contralateral tingling and or numbness, ${ }^{23}$ which may then spread along adjacent body parts as the seizure propagates through the sensory homunculus. Some patients also describe pain or altered thermal sensation. The seizure may propagate to the frontal lobe resulting in clonic motor activity often with tonic posturing.

\section{Parietal association areas}

In seizures arising from the non-dominant parietal association cortex, patients may describe distortions of body image. Others describe sensations of twisting or turning sensations. ${ }^{23}$ In seizures arising from the superior parietal lobule and precuneus, there may be altered body perception such as macrosomatognosis (body part being enlarged) or microsomatagnosis (body part being shrunken), particularly of the distal extremities. ${ }^{26}$ Other visual illusions include epileptic kinetopsia (illusion of movement of a stationary object) in the superior parietal lobule and intraparietal sulcus, ${ }^{27}$ and macropsia (objects appearing larger) or micropsia (objects appearing smaller) in the precuneus. ${ }^{25}$ Subjective vertiginous sensations may occur in seizures arising from various parts of the parietal lobe. Autoscopic phenomena can occur in both temporal and parietal lobe epilepsies and often implicate the temporoparietal junction and vestibular cortex. In the dominant parietotemporal region, there may be language impairment with difficulties reading and writing. Seizures arising from the secondary sensory area, in addition to poorly localised sensory aura affecting distal extremities and face, may involve a feeling of inability to move the contralateral limbs.

Parietal lobe seizures may propagate in different ways resulting in variable clinical manifestations. Those that propagate to sensorimotor cortex may show focal motor clonic activity contralateral to the epileptogenic zone; those propagating to the supplementary motor area may have tonic posturing of the limbs; and those propagating to the temporal region may develop temporal aura or automatisms. Seizures from more superior areas of the parietal lobe appear to spread preferentially to the frontal lobe while seizures from more inferior regions of the parietal lobe appear to propagate to temporal areas. 


\section{OCCIPITAL LOBE SEIZURES}

Seizures arising from the occipital lobes are usually (in $40 \%-75 \%$ of patients) characterised by visual aura, or less commonly by oculomotor features, such as eye deviation, blinking or nystagmus. However, their seizure semiology may also reflect propagation to temporal or frontal lobes. ${ }^{28} 29$

\section{Primary visual cortex}

Seizures arising from primary visual (calcarine) cortex cause an elementary visual aura, and tend to be brief, usually less than $2 \mathrm{~min}$, and thus are shorter than a typical migrainous aura. Patients may describe flashing coloured or bright white lights, often spherical, but they may take different forms and shapes. The discharges originating in the lateral occipital cortex probably produce the pulsating quality of the visual features. In most patients, the visual phenomena are lateralised and contralateral to the hemisphere of seizure onset; however, up to $30 \%$ have more diffuse visual changes. ${ }^{30}$ True ictal visual field loss or amaurosis is rare, and may represent a postictal phenomenon, particularly if long lasting.

\section{Visual association areas}

Complex visual hallucinations may occur in occipital lobe epilepsy but are less common than elementary phenomena. They occur when seizures involve the prestriate cortex or may indicate propagation to adjacent temporo-parietal areas. Patients may report complex formed visual hallucinations connected with past events, or kinetopsia, macropsia or micropsia. Rarely, patients report autoscopy, perceiving mirror images of themselves.

\section{Parieto-occipital junction}

Oculomotor manifestations may occur, including eye version, blinking and nystagmus. In a large series, ${ }^{30}$ $20 \%$ of patients had eye blinking at seizure onset. Although eye version may represent propagation, in two reported cases this occurred while ictal discharges were localised to occipital lobe. Epileptic nystagmus may also develop, typically with the fast phase to the contralateral hemisphere and the slow component to the ipsilateral side. ${ }^{31}$

\section{CINGULATE GYRUS}

The cingulate gyrus forms part of the limbic network; it consists of anterior, middle and posterior parts, and has extensive functional connectivity with other brain regions. Most data on semiology of seizures are from case series of lesional cases. Seizures arising from the anterior cingulate are characterised by intense fright, screaming and vocalisations, with autonomic signs and complex gestural automatisms or hypermotor activity. ${ }^{32}$ A later case series ${ }^{33}$ that included cases with seizures arising from both posterior and anterior cingulate, identified that there were two groups of patients with seizures arising from the anterior cingulate: those with typical hypermotor seizures with fear, autonomic features and infrequent generalisation, and a subgroup (all with infiltrating astrocytomas) with simple motor features and frequent generalisation. In this series, all those whose seizures arose from the posterior cingulate had symptoms suggesting temporal lobe epilepsy with auras (eg, psychic, abdominal and gustatory). However, another study ${ }^{34}$ reported that seizures arising from the posterior cingulate showed semiology that varied depending on their spread: those spreading to frontal areas showed motor manifestations-including bilateral asymmetric tonic seizures and hypermotor seizures-while those spreading to temporal areas showed dialeptic seizures (that is seizures where the main feature is loss of awareness and behavioural arrest) or automotor seizures. Gelastic seizures may also arise from the anterior cingulate as well as 'chapeau de gendarme' (down turning of the mouth); these are discussed separately below. In summary, seizures arising from the anterior cingulate tend to present with typical features, whereas those from the posterior cingulate show variable features depending on propagation patterns.

\section{INSULAR SEIZURES}

The insular lobe has two parts, a larger anterior part and a smaller posterior part, and is highly connected with several other brain regions, integrating information required for cognitive, behavioural, and sensory processing. ${ }^{35}$ Seizures arising from the insular lobe are rare, but have heterogenous semiology that include viscerosensory, somatosensory, autonomic symptoms (nausea, hypersalivation, piloerection, heart rate changes), language disturbance, complex auditory aura and (due to propagation to frontal and temporal areas) automotor and hypermotor behaviours, tonic and/or clonic motor manifestations. ${ }^{36}$ This variability along with often non-localising EEG due to deep location, can mean that clinicians may not recognise such seizures as arising from the insula.

Some studies have suggested that viscerosensory symptoms-such as abdominal aura and laryngeal constriction, and autonomic signs, including nausea, hypersalivation, and sweating - arise from the anterior insula whereas painful sensations-including burning, electric shock and tingling-more likely arise from the posterior insula. ${ }^{37}$ Non-painful somatosensory aura can also occur. Stimulation studies have identified several sensory features that help to differentiate insular seizures from seizures arising from primary and secondary somatosensory cortex: insular responses tend to affect a larger skin territory including perioral regions, can be bilateral or ipsilateral, and more likely evolve to complex motor phenomena.

Seizures arising from hippocampus almost always propagate to the insula; thus an abdominal aura is 


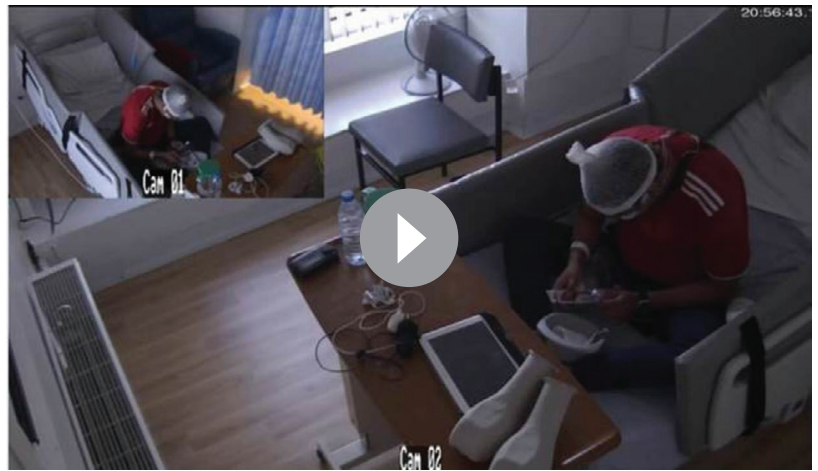

Video 5 Video case 5

also common in mesiotemporal seizures. Due to rapid propagation, insular seizures can mimic seizures arising from the temporal or frontal lobe. Nevertheless, the combination of complex visceral and somatosensory auras points towards an insular onset.

\section{SPECIAL SEIZURES}

\section{Gelastic seizures}

Gelastic seizures typically arise from hypothalamic hamartomas. However, the act of laughing is part of a complex network involving cingulate, frontal and temporal regions and so laughing also occurs in seizures arising from other regions. In a case series from our centre, ${ }^{14}$ a third of cases had hypothalamic hamartomas, whereas a third of seizures were temporal, and a third frontal.

- Gelastic seizures arising from hypothalamic hamartomas usually present in early childhood, are brief, stereotyped and very frequent (video 5). The laughter is usually mirthless and without impaired consciousness, but autonomic signs, such as facial flushing and pupillary dilation, are common. There may also be a dacrystic (crying) component.

- Gelastic seizures arise from brain structures other than the hypothalamus usually present in adulthood. Those in temporal lobe epilepsy are usually part of more complex seizure semiology with automotor features and loss of consciousness.

Video case 5: An 18-year-old right-handed man had onset of gelastic seizures from aged 2 years. Imaging confirmed a hypothalamic hamartoma (figure 6). Videotelemetry captured typical gelastic seizure, without mirth, though he lost awareness in some of his seizures.

\section{Chapeau de gendarme}

The 'chapeau de gendarme' or ictal pout sign is characterised by a downturned mouth from bilateral lip and chin contraction. ${ }^{38}$ If seen early in the seizure it is highly localising to frontal lobe onset, in particular anterior cingulate, orbitofrontal region or mesioprefrontal or premotor cortex (video 6). ${ }^{39}$

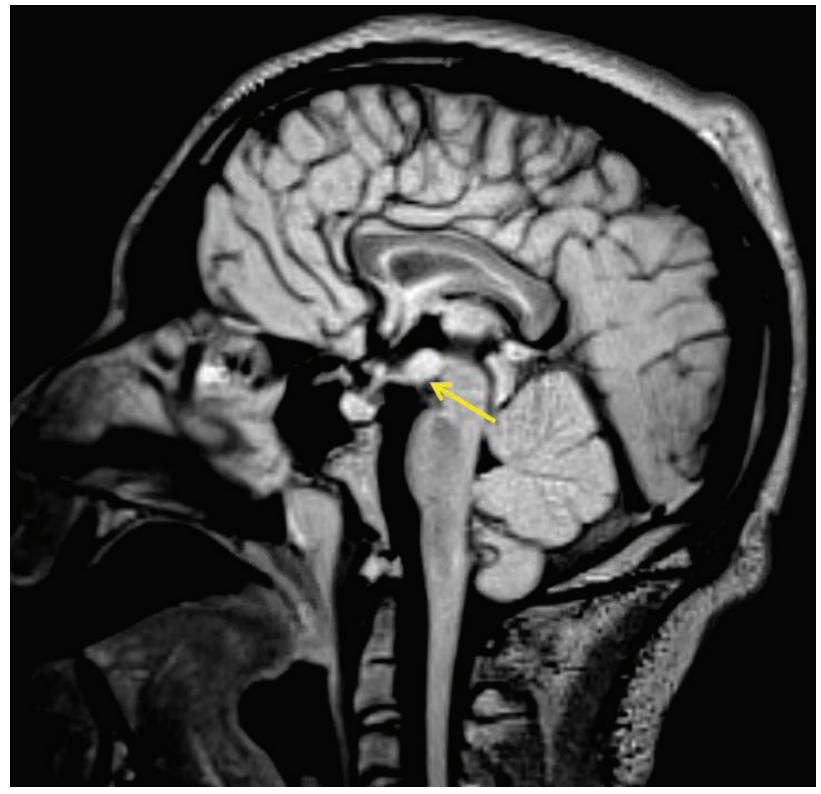

Figure 6 Case 5: Fluid attenuated inversion recovery (FLAIR) MR scan of brain showing hypothalamic hamartoma (shown with arrow).

Video case 6: A 20-year-old man had onset of seizures at aged 12 years. He had seizures with motor features and loss of awareness indicating propagation to frontal areas. MR scan of brain was nonlesional. Videotelemetry captured seizures involving behavioural arrest and then facial grimacing with downturned mouth (chapeau de gendarme) along with other motor features including rocking, chewing and head movement. Ictal EEG was non-localising.

\section{Reflex seizures}

Reflex seizures are triggered by specific stimuli. ${ }^{40}$ Photosensitive epilepsy is the most common reflex epilepsy; although more commonly associated with idiopathic (genetic) genetic generalised epilepsy, this also occurs in some focal epilepsies, where it suggests an occipital onset. Other reflex focal epilepsies are rare but may also have localising value. For example:

- Musicogenic seizures localise to the temporal lobe and can occur in both mesial and neocortical temporal epilepsies.

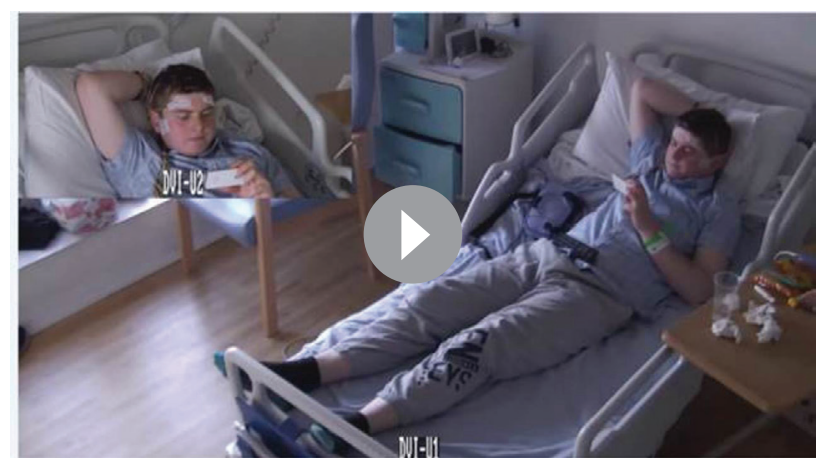

Video 6 Video case 6. 


\begin{tabular}{ll}
\hline Table 3 & Localising value of auras \\
\hline Aura & Localisation \\
\hline Somatosensory & \\
\hline $\begin{array}{l}\text { Marching sensory aura } \\
\text { Tingling, numbness }\end{array}$ & $\begin{array}{l}\text { Primary sensory cortex } \\
\text { Primary or secondary somatosensory } \\
\text { cortex, supplementary motor area, insula }\end{array}$ \\
\hline $\begin{array}{l}\text { Pain/ warmth } \\
\text { Visual aura }\end{array}$ & Secondary somatosensory cortex, insula \\
\hline $\begin{array}{l}\text { Elementary (lights, } \\
\text { shapes) }\end{array}$ & Primary visual cortex \\
\hline Illusions/ distortions & Temporo-parieto-occipital \\
\hline $\begin{array}{l}\text { Complex visual } \\
\text { hallucinations }\end{array}$ & Temporal \\
\hline Auditory & \\
\hline Elementary & Heschl's gyrus, superior temporal \\
\hline Illusions/distortion & Lateral temporal, insula \\
\hline $\begin{array}{l}\text { Other } \\
\text { Gustatory } \\
\text { Olfactory }\end{array}$ & Insula, mesiotemporal \\
\hline Vestibular & Insula, mesiotemporal, orbitofrontal \\
\hline Autonomic & Posterior temporal, parietal \\
\hline & Insula, amygdala, cingulate \\
\hline
\end{tabular}

- Startle-induced tonic or atonic seizures triggered by auditory stimuli localise to onset in or near supplementary motor area.

- Eating-induced seizures usually arise from temporal or extratemporal perisylvian area.

In these epilepsies, spontaneous seizures can also occur.

\section{AURA}

This is often the first ictal symptom, and therefore can provide useful localising information. Table 3 summarises various aura types .

\section{LATERALISING SIGNS}

As well as helping with localisation, some signs have lateralising value. Robust lateralising motor signs (positive predictive value of $>80 \%$ ) already discussed include unilateral clonic movements, unilateral tonic or dystonic posturing and early head version: these are

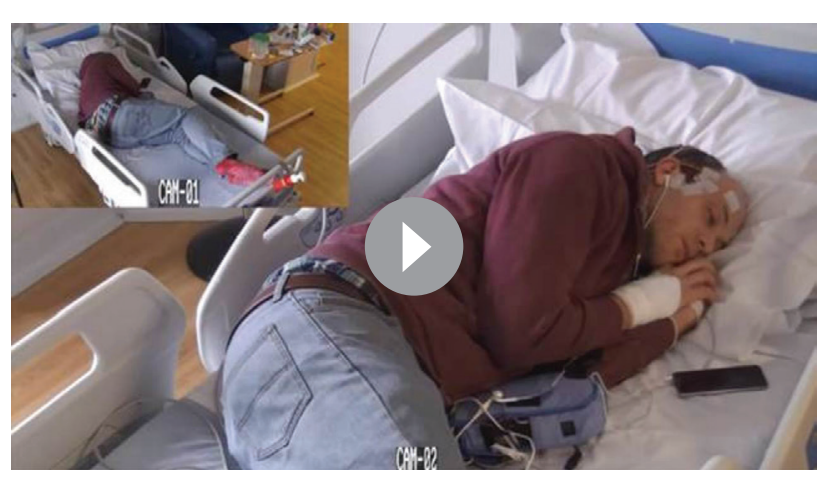

Video 7 Video case 7 clinical signs contralateral to the side of onset. Other lateralising motor phenomena are a 'figure-of-4' sign before secondary generalisation (video 7) where the extended arm is contralateral to seizure onset, there is asymmetric clonic ending of generalised phase with the last clonic jerk ipsilateral to seizure onset, and postictal Todd's paresis contralateral to seizure onset. ${ }^{20}$ These features do not differentiate between frontal or extrafrontal onset. Unilateral sensory aura is a contralateral sign. $^{23}$

Video case 7: A 33-year-old man had seizure onset at age 21 years, starting with psychic/gustatory aura and evolving to an automotor seizures with some focal to bilateral tonic-clonic seizures. MR scan of brain was non-lesional and PET scan showed left temporal hypometabolism. Videotelemetry captured two habitual seizures with automotor features evolving to bilateral tonic-clonic seizures. Before generalisation, there was head version (forced, sustained and extreme turning of the head) to the right, followed by a 'figureof-4' with right arm extension, indicating left hemispheric onset. In one of the seizures, the last clonic jerk was on the left, which also pointed to left hemispheric onset. Ictal EEG was non-localisable in one but lateralised to the left in another.

Ictal or postictal dysphasia lateralises to dominant hemisphere but is of poor localising value and has to be differentiated from speech arrest which is nonlateralising. Ictal speech-speech with formed nonsensical phrases-is a non-dominant sign. Preserved awareness during ictal automatism points to the nondominant hemisphere. ${ }^{15}$ Rhythmic ictal non-clonic hand motions may occur as a contralateral sign in temporal lobe epilepsy. ${ }^{41}$ A simple auditory aura, if unilateral (which is rare) is a contralateral sign. Other lateralising signs in temporal lobe epilepsy include peri-ictal drinking, ictal spitting, ictal vomiting and an urge to urinate, which point to a non-dominant focus.

Hemifield visual aura has good lateralising value to the contralateral occipital lobe. ${ }^{30}$ Unilateral eye blinking lateralises to the hemisphere ipsilateral to the eye-blinking. ${ }^{42}$ In epileptic nystagmus, the fast component is to the side contralateral to hemisphere of seizure onset. ${ }^{21}$

\section{CONCLUSION}

Careful analysis of seizure semiology can help to localise seizures, which is particularly important when evaluating people for epilepsy surgery. Identifying seizure semiology requires a meticulous history to ascertain details of auras, and careful analysis of seizures captured during video-EEG recordings.

Many seizure symptoms and signs have robust localising and lateralising value. However, seizure localisation can still be challenging for several reasons, including the fact that seizure semiology may become apparent only once a seizure has spread to an eloquent area or has sufficiently engaged brain networks. 


\section{Key points}

Seizure semiology reflects altered electrical activity in the affected part of the brain.

- Seizures can manifest as motor, subjective and behavioural phenomena, or loss of awareness, and careful analysis of their semiology can allow localisation of seizure onset.

- Ictal manifestations may represent propagation of the epileptic discharge from its origin.

\section{Further reading}

1. Noachtar S, Peters AS. Semiology of epileptic seizures: A critical review. Epi and Behav 2009; 15: 2-9

2. Foldvary-Schaefer $N$, Ungewanse K. Localizing and Lateralizing features of aura and seizures. Epi and Behav 2011; 20: 160-166

Moreover, its semiology may relate not only to activation of brain areas but also to disruption of brain function and disinhibition of networks that mediate complex behaviours. Nevertheless, careful interpretation of seizure semiology enhances seizure localisation, particularly when collated with other information including ictal EEG activity, structural and functional imaging, and neuropsychometry.

Acknowledgements With thanks to Dr Kelly Pegoretti Baruteau, Consultant Neuroradiologist, for her help with selecting MR images for this manuscript, and to the Videotelemetry Team at the National Hospital for Neurology and Neurosurgery for their contribution to acquiring the Videotelemetry data.

Contributors FAC drafted and edited the manuscript. RS helped with the literature review and collection of video cases. MCW provided critical review of the manuscript. BW contributed to figures and editing the article.

Funding The authors have not declared a specific grant for this research from any funding agency in the public, commercial or not-for-profit sectors.

Competing interests None declared.

Patient consent for publication Not required.

Provenance and peer review Commissioned; externally peer reviewed by Khalid Hamandi, Cardiff, UK.

\section{ORCID ID}

Fahmida A Chowdhury http://orcid.org/0000-0001-9546-0197

\section{REFERENCES}

1 Eadie MJ, Bladin PF. Disease once sacred: a history of the medical understanding of epilepsy, 2001.

2 Rosenow F, Lüders H. Presurgical evaluation of epilepsy. Brain 2001;124:1683-700.

3 Foldvary-Schaefer N, Unnwongse K. Localizing and lateralizing features of auras and seizures. Epilepsy Behav 2011;20:160-6.
4 Liou J-Y, Smith EH, Bateman LM, et al. A model for focal seizure onset, propagation, evolution, and progression. Elife 2020;9:e50927.

5 Elwan S, Alexopoulos A, Silveira DC, et al. Lateralizing and localizing value of seizure semiology: comparison with scalp EEG, MRI and PET in patients successfully treated with resective epilepsy surgery. Seizure 2018;61:203-8.

6 Bonini F, McGonigal A, Trébuchon A, et al. Frontal lobe seizures: from clinical semiology to localization. Epilepsia 2014;55:264-77.

7 McGonigal A, Chauvel P. Prefrontal seizures manifesting as motor stereotypies. Mov Disord 2014;29:1181-5

8 McGonigal A, Chauvel P. Frontal lobe epilepsy: seizure semiology and presurgical evaluation. Pract Neurol 2004;4:260-73.

9 Ikeda A, Sato T, Ohara S, et al. "Supplementary motor area (SMA) seizure" rather than "SMA epilepsy" in optimal surgical candidates: a document of subdural mapping. J Neurol Sci 2002;202:43-52.

10 Werhahn KJ, Noachtar S, Arnold S, et al. Tonic seizures: their significance for lateralization and frequency in different focal epileptic syndromes. Epilepsia 2000;41:1153-61.

11 Sitthinamsuwan B, Usui N, Tottori T, et al. Seizures with tonic posturing: Semiologic difference between supplementary sensorimotor area (SSMA) origin and extra-SSMA origin. Epilepsia 2016;57:e39-44.

12 Chibane IS, Boucher O, Dubeau F, et al. Orbitofrontal epilepsy: case series and review of literature. Epilepsy Behav 2017;76:32-8.

13 Ikeda A, Hirasawa K-ichi, Kinoshita M, et al. Negative motor seizure arising from the negative motor area: is it ictal apraxia? Epilepsia 2009;50:2072-84.

14 Kovac S, Diehl B, Wehner T, et al. Gelastic seizures: incidence, clinical and EEG features in adult patients undergoing videoEEG telemetry. Epilepsia 2015;56:e1-5.

15 Blair RDG. Temporal lobe epilepsy semiology. Epilepsy Res Treat 2012;2012:1-10.

16 Henkel A, Noachtar S, Pfänder M, et al. The localizing value of the abdominal aura and its evolution: a study in focal epilepsies. Neurology 2002;58:271-6.

17 Erickson JC, Clapp LE, Ford G, et al. Somatosensory auras in refractory temporal lobe epilepsy. Epilepsia 2006;47:202-6.

18 Maillard L, Vignal J-P, Gavaret M, et al. Semiologic and electrophysiologic correlations in temporal lobe seizure subtypes. Epilepsia 2004;45:1590-9.

19 Du X, Usui N, Terada K, et al. Semiological and electroencephalographic features of epilepsy with amygdalar lesion. Epilepsy Res 2015;111:45-53.

20 Marashly A, Ewida A, Agarwal R, et al. Ictal motor sequences: lateralization and localization values. Epilepsia 2016;57:369-75.

21 Chabardès S, Kahane P, Minotti L, et al. The temporopolar cortex plays a pivotal role in temporal lobe seizures. Brain 2005;128:1818-31.

22 Wang L, Mathews GC, Whetsell WO, et al. Hypermotor seizures in patients with temporal pole lesions. Epilepsy Res 2008;82:93-8

23 Salanova V. Parietal lobe epilepsy. J Clin Neurophysiol 2012;29:392-6.

24 Asadollahi M, Sperling MR, Rabiei AH, et al. Drug-Resistant parietal lobe epilepsy: clinical manifestations and surgery outcome. Epileptic Disord 2017;19:35-9. 
25 Francione S, Liava A, Mai R, et al. Drug-Resistant parietal epilepsy: polymorphic ictal semiology does not preclude good post-surgical outcome. Epileptic Disord 2015;17:32-46.

26 Yang Y, Wang H, Zhou W, et al. Electroclinical characteristics of seizures arising from the precuneus based on stereoelectroencephalography (SEEG). BMC Neurol 2018;18:110.

27 Perumal MB, Chinnasami S, Shah A, et al. Epileptic kinetopsia localizes to superior parietal lobule and intraparietal sulcus. Neurology 2014;83:768-70.

28 Blume WT, Wiebe S, Tapsell LM. Occipital epilepsy: lateral versus mesial. Brain 2005;128:1209-25.

29 Kuzniecky R. Symptomatic occipital lobe epilepsy. Epilepsia 1998;39(Suppl 4):S24-31.

30 Salanova V, Andermann F, Olivier A, et al. Occipital lobe epilepsy: electroclinical manifestations, electrocorticography, cortical stimulation and outcome in 42 patients treated between 1930 and 1991. surgery of occipital lobe epilepsy. Brain 1992;115(Pt 6):1655-80.

31 Lee S-U, Suh H-I, Choi JY, et al. Epileptic nystagmus: a case report and systematic review. Epilepsy Behav Case Rep 2014;2:156-60.

32 Bancaud J, Talairach J. Clinical semiology of frontal lobe seizures. Adv Neurol 1992;57:3-58.
33 Alkawadri R, So NK, Van Ness PC, et al. Cingulate epilepsy: report of 3 electroclinical subtypes with surgical outcomes. JAMA Neurol 2013;70:995-1002.

34 Enatsu R, Bulacio J, Nair DR, et al. Posterior cingulate epilepsy: clinical and neurophysiological analysis. J Neurol Neurosurg Psychiatry 2014;85:44-50.

35 Isnard J, Guénot M, Sindou M, et al. Clinical manifestations of insular lobe seizures: a stereo-electroencephalographic study. Epilepsia 2004;45:1079-90.

36 Peltola ME, Trebuchon A, Lagarde S. Anatomoelectroclinical features of SEEG-confirmed pure insular-onset epilepsy. Epilepsy Behav 2020;21:105.

37 Jobst BC, Gonzalez-Martinez J, Isnard J, et al. The insula and its epilepsies. Epilepsy Curr 2019;19:11-21.

38 Hayakawa I, Kubota M. Ictal pouting: Kabuki visage or chapeau de gendarme? Pract Neurol 2018;18:410-2.

39 Tan Y-L, Muhlhofer W, Knowlton R. Pearls and Oy-sters: the chapeau de gendarme sign and other localizing gems in frontal lobe epilepsy. Neurology 2016;87:e103-5.

40 Italiano D, Ferlazzo E, Gasparini S, et al. Generalized versus partial reflex seizures: a review. Seizure 2014;23:512-20.

41 Lee GR, Arain A, Lim N, et al. Rhythmic ictal nonclonic hand (RINCH) motions: a distinct contralateral sign in temporal lobe epilepsy. Epilepsia 2006;47:2189-92.

42 Benbadis SR, Kotagal P, Klem GH. Unilateral blinking: a lateralizing sign in partial seizures. Neurology 1996;46:45-8. 